Die vorliegende Nummer erscheint auf 28 Textseiten verstärkt. Dafür wird die nächste Nummer nur 20 Seiten umfassen. Aus technischen Gründen soll dieser Wechsel des Umfanges bis auf weiteres beibehalten werden.

Eine kaukasisch-balkanische Beziehung? Von Ernst Le wy.

Wer vom indogermanischen Standpunkte an das Armenische herangeht, den überrascht am meisten in dieser Sprache, dem macht am schwersten die Eingewöhnung in sie jener eigentümliche Gebrauch der postponierten "Artikel ${ }^{4}$ s, d, n, der diese Elemente außerhalb" jeder Kongruenz stellt (z. B. patasxani et hreštak-n ev ase c-kanajs-n Math. 28, 5, Meillet Altarm. El.

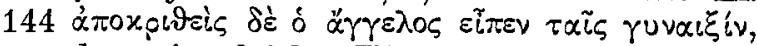
wo also $n$ in gleicher Weise den Singular: der Engel, wie den Plural: den Frauen hervorhebt) und sogar, was merkwürdigerweise gar nicht hervorgehoben zu werden scheint, auch an Verba anhängt (z. B. k'anzi jareaw, orpès asacn-

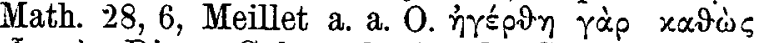
$\varepsilon i \pi \varepsilon v$.) Dieser Gebrauch gibt der Sprache einen hervorragend demonstrativen Charakter, wie man ihn auch in einer im Kaukasus angesiedelten idg. Sprache erwarten darf: der deutende, wiederaufnehmende Charakter einheimischer Sprachen des Kaukasus ist durch Heinrich Winkler's Arbeiten bekanntgeworden. Doch bleibt auffallend die eigentümliche Dreiheit der Elemente: $\mathrm{s}, \mathrm{d}, \mathrm{n}^{\mathrm{1}}$.

Es ist ein großes Verdienst Brugmanns, daß er (Demonstrativpron. 45) die Parallele zu dieser armenischen Erscheinung aus bulgarischen Dialekten nachgewiesen hat. Wir finden da nämlich nicht nur den einen suffigierten Artikel der bulgarischen Schriftsprache, sondern drei Elemente $s, t, n$ (neben $s$ steht allerdings auch $v$ in gleicher Bedeutung) im Sinne von hier, da, dort", wie im Arm². Brugmann erklärt diese bulg. Eigenheit als einen Armeniacismus, $v 0 n$ derselben Art, wie so manche von den Gräcis-

1) $\mathrm{DaB}$ diese eine Art Umsetzung des ja sonst im Arm. ganz geschwundenen idg. Genusunterschiedes sei, auf diesen Gedanken konnte man vielleicht kommen. Wir sind über die formalen Vorgänge bei der Sprachveränderung noch so wenig orientiert, daß ich diesen mir unwahrscheinlichen Gedanken doch nicht ganz, mit einer eingängigen Begründung, ablehnen kann.

2) Allerdings wohl nicht im Anschlu $\beta$ an Verba gebraucht. Ich urteile nur auf Grund der von Brugmann angeführten Beispiele, da mir dialektische Texte für das Bulg. nicht zur Verfügung stehen. 145 men im Lat., Gallicismen im Deutschen, Germanismen im Lit. usw." durch den lebhaften Verkehr mit den auch in Bulgarien angesiedelten Armeniern. $\mathrm{Ob}$ wir den Armeniern die zu diesem Einfluß nötige höhere Kultur zuschreiben dürfen, weiß ich nicht; aber - und das ist wichtiger kann in einem der von Brugmann erwähnten Fälle nachgewiesen werden, daß da der Sprachbau, die Form der Sprache so bedeutend beeinflußt worden wäre? Selbst im Magyarischen, das von Germanismen geradezn wimmelt, kann man kaum einen Germanismus nachweisen, der irgendwie den Sprachbau berührt. Aus dem Rumänischen kann, wie Brugmann sehr richtig bemerkt (a. a. 0. 44), der bulg. Artikel nicht entlehnt sein; wie weit und von wem die Ansicht von Kopitor und Miklosich, daß der bulg. und rumän. Artikel thrako-illyrischem Einfluß sein Dasein verdanke" ,heute überwunden" ist, entzieht sich meiner Kenntnis; ein richtiger Kern könnte darin stecken.

Das eine können wir zunächt sagen, daß diese Eigenheit des Bulgarischen weder alt-idg. noch uralaltaiisch ${ }^{1}$ ist. (Das Hervortreten uralaltaiischer Spracheigentümlichkeiten dürften wir ja in der Sprache der Bulgaren erwarten, da sie ein türkisches Volk sind.) Da diese Eigenheit aber in ihrer Sprache auftritt, und wenn, was $m$. A. n. der Fall ist, sie nicht aus armenischem Einfluß hergeleitet werden kann, müßte sie, wofern meine mehrfach zitierte Ansicht über die Sprachmischung richtig ist, (s. OLZ. 1922. (Sp. 133) altbalkanisch sein.

Wo wohnen heute die Bulgaren? In der Gegend, wo auch die Bryger gewohnt haben. Daß die Bryger zu den Phrygern gehören, und daß diese die nächsten Verwandten der Armenier

1) Auf uralaltaijschem Gebiet haben, soviel ich weiß nur die Mordwinen einen postponierten Artikel entwickelt. Diese Eigenheit des Mordwinischen ist natürlich nur im Zusammenhange des ganzen Baues dieser Sprache verständlich, und beruht, wie ich allerdings meine, auch auf fremdem, vielleicht kaukasischem Einfluß; s. darüber mein "Zum Bau des Erdsja-Mordwinischen" (Mellrichstadt 1921; i. K. bei Priebatsch's Buchhandlung, Breslau). Die semitischen Verhäl tnisse kann ich leider gar nicht beurteilen; sonderbar ist es doch aber wirklich, daß gerade im Aramäischen ein postponierter Artikel existiert. 
seien, wird doch überliefert. Dann wäre die erwähnte Verwendung der drei Demonstrativstämme als eine altbalkanische, im (Phrygisch-)Armenischen wie in bulgarischen Dialekten wieder zu Tage tretende und durchgeführte Eigenheit anzusehen, deren Entwicklung in Armenien der kaukasische Einfluß vielleicht noch begünstigt hat.

Vielleicht liesse sich eine Deutung für die hier erwähnten Dinge noch in anderer Richtung ausdenken; ich möchte aber meinen, daß es sich lohne, in der angedeuteten zu suchen.

\section{Offener Brief an den Herausgeber.}

Sehr verehrter Herr Kollege!

Mit großem Interesse habe ich die Abhandlung Hermann Schneiders über die neuentdeckte Sinaiinschrift in OLZ $1921 \mathrm{Nr} .11$ gelesen. Es ist vielleicht nicht unbescheiden, darauf hinzuweisen, daß in den Sitzungsberichten der Bayerischen Akademie der Wissensch., Philos.philol. Klasse, 1920 eine die Datierung der Petrieschen Sinaiinschriften ïberschriebene Abhandlung erschienen ist, die in ähnlicher Weise wie Dr. Schneider an der bisher üblichen hohen Ansetzung und an der Zuverlässigkeit der Eislerschen Lesungen Kritik übte (die Inschriften sind jünger als 1500 v. Chr.; um wieviel bleibt zunächst unentschieden, doch spricht vieles dafür, nicht unter das letzte Drittel des zweiten vorchristlichen Jahrtausends hinabzugehen). Auch das ist dort schon ausgesprochen, daß die Sinaiinschrift eine Verwandte, aber anch kaum mehr, der phönikischen Schrift ist, von einem Mann erfunden, der nur eine oberflächliche Kenntnis der Hieroglyphen und ihrer Möglichkeit, Einzelbuchstaben auszudrücken, hatte. Diese Beziehungen zur ägyptischen Schrift ganz zu leugnen, geht allerdings meiner Meinung nach zu weit. Beziehungen zu den bisher'bekannten ,kretischen" Schriftarten scheinen mir noch fraglicher.

Ich bitte, die Gelegenheit zu einer Feststellung benutzen zu dürfen, auf die Heinrich Schäfer zunächst meine Aufmerksamkeit gelenkt hat. Auch er war der Meinung, daß der Typus der kubischen Hockfigur nicht vor dem Anfang der XVIII. Dvnastie nachweisbar sei. Aber bei Budge, A guide to the Egyptian collections 1909, Taf. XXIV, S. 215 ist eine unter Amenemes II. datierte Stele abgebildet, in deren Türvertiefung eine hockende Statue sitzt, deren Inschrift (Hieroglyphic texts from Egyptian stelae II, Taf. 19, 20) keinen Zweifel läßt, daß derselbe Mann oder allenfalls sein Sohn dargestellt ist. Leider wissen wir über die Herkunft des aus Sammlung Anastasi stammenden Stückes nichts. Es beweist aber, daß vereinzelt der Typus der berühmten Statuen des Senmut und Bekenchons schon im
Mittleren Reich vorkam, daß also in diesem Punkt meine Ausführungen einer gewissen Einschränkung bedürfen. Immerhin bleibt wenig wahrscheinlich, daß der damals zweifellos sehr seltene Typus schon auf dem Sinai heimisch geworden sein sollte.

Mit besten Grüßen

$$
\mathrm{Ihr}
$$

Fr. W. von Bissing.

\section{Besprechungen.}

Rotter, Gough,v.Buttel-Reepen, Armbruster: Die Biene in Ägypten jetzt und yor 5000 Jahren. Mit 3 Tafeln und 4 Textabbldgen. (Archir f. Bienenkunde. Hrsg. v. Dr. L. Armbruster III. Jahrg., 1. und .. Heft.) (82 S.) gr. 80. Freiburg, Th. Fisher 1921. M. 14. - Bespr. $\nabla$. Waiter Wreszinski-Königsbg. i. Pr.

Von den 4 in dem Heft vereinigten Aufsätzen kommt für die Leser der OLZ eigentlich nur der letzte (S. 68-80), der von Armbruster, in betracht, worin nachgewiesen wird, daß die Bienenhaltung im Ágypten des $A R$ und dem von heute die gleiche ist. A. behandelt darin das Berliner Relief 20037 aus der Kammer der Jahreszeiten in Abu Gurab, das auf der linken, zerstörten Seite vielleicht die Reste eines Bienenstandes enthält, der mit den heute üblichen übereinstimmen könnte, die aus $1 \mathrm{~m}$ langen, übereinander geschichteten röhrenförmigen Krügen bestehen. Die bei der Ernte beschäftigten Männer tun, was die darüberstehenden Inschriften besagen: sie „blasen " Rauch gegen einen Stock, um die Bienen zu vertreiben und den Stock entleeren zu können, füllen ${ }^{*}$ die Waben in einen Krug und füllen den ausgelaufenen Honig in ein anderes Gefäß um (dies letztere nach Ansicht des Ref., doch ist beides unsicher), „brauen" Honigbier und siegeln" die gefüllten Honigkrüge. Der Verf. scheint in der Hauptsache gegen die Deutungen von Luise Klebs Recht zu behalten; wo er aus den Darstellungen noch genauere Einzelheiten zu erschließen gesucht hat, gerät er freilich in die Irrtümer, denen jeder verfällt, der die ägyptischen Bilder zu „wörtlich" nimmt. - Bemerkenswert ist seine Feststellung, daß die Hieroglyphe gerade wegen der gelben Farbe keine Hornisse, sondern die Honigbiene Apis mellifica fasciata darstellt. -

0 w, A. Frhr. $\nabla .:$ Joseph 7. Agspten u. Aseneth. (III, 169 S.) 80. Regensburg, Vlgsh. 7. Manz 1918. M. 4.20 Bespr. von G. Möller †, Berlin.

Der alte Jesuitenpater Athanasius Kircher wïrde seine helle Freude an diesem phantasievollen Büchlein seines Amtsgenossen gehabt haben. Mehr vielleicht noch der selige Joseph Lauth, der Verfasser des „Moses-'Hosarsyphos-Sali-Hus Levites-A'haron frater, Ziphorak-Debariah conjux, Miriam-Bellet soror, Elisheba-Elizebat fratria, ex 